\title{
FEATURES OF OPTICAL MODELING IN EDUCATIONAL AND SCIENTIFIC ACTIVITY
}

\author{
I. I. Bayneva
}

Associate Professor of the Department of Lighting equipment at the Institute of Electronics and Lighting equipment of the Mordovian State University named after N.P. Ogarev

Published online: 15 February 2017

\begin{abstract}
The article discusses the functionality of existing software for the modeling, analysis and optimization of lighting systems and optical elements, through which the stage of their design can be automated completely. The use of these programs is shown using the example of scientific work and the educational activity of masters.
\end{abstract}

Key words: lighting device, modeling, design, software, optical system, automation, educational activity.

\section{INTRODUCTION}

The design and production of modern optical systems requires the modeling of complex physical phenomena. Light propagation models in optical elements make the part of optical modeling systems. Such systems provide end-to-end modeling, that is, the propagation of light from the sources to radiation receivers.

The design of modern optical systems, such as mirror-lens lighting systems, liquid-crystal display systems, multi-lens lenses, LEDs, etc., requires the use of physically accurate and efficient models of light propagation within these devices. However, the use of such propagation models is necessary, but not a sufficient condition for an effective design. A sufficient condition is a visual representation of the data obtained in the process of optical modeling. In the field of optics and optical instrument engineering, specialized CAE systems (systems of engineering analysis) are used,

Author Correspondence, e-mail: author@gmail.com

doi: http://dx.doi.org/10.4314/jfas.v8i2s.638 
each of which allows to solve a certain design problem [1,2]. There are several dozens of universal software packages among them to design the optical systems for various purposes and a number of systems used for the design of specialized systems.

\section{Optical system models}

There are many options for non-pictorial optical system modeling, however, it is more convenient to use mathematical and computer modeling.

The mathematical models of an optical system may be represented by the models of geometric optics, which make the basis for a light beam passage modeling, and an optical analysis is used to calculate the trajectory of rays. The construction and the study of models facilitate the study of the properties and regularities available in a real device and often saves money on a prototype production. This model is being developed to study the given properties and improve the characteristics of an optical system.

Any model of an optical system must have the following properties:

- adequacy - the correspondence of an optical system model to the initial real system taking into account the parameters and technical characteristics;

- accuracy - the degree of results coincidence obtained during the modeling of optical system results with pre-established and desired ones;

- universality - the applicability of an optical system model to the analysis of a number of similar systems in one or several different design problems.

the simulation of a light source is an integral part of a lighting system modeling. Modern software packages designed to calculate non-imaging optics have several methods to model radiation sources.

The first method is the use of simple built-in source models and the construction of more complex light sources on their basis. A separate beam, a point source, a Lambertian flat radiator and a luminous spiral can be considered as integrated models. On the basis of a flat radiator model, almost any model can be designed, that is, to set a complex light source by element modeling of its parts that influence the motion of rays. During complex source designing, this method is quite laborious, since the information about such a source is necessary (design parameters of a system). Such information is not always possible to obtain.

Therefore, another method is used often - the modeling with a file that already contains the information about the radiation at a light source output. Very often large manufacturers of optical lighting systems create models in the format supported by the programs for illumination and optics calculation, which can be obtained from manufacturer's websites. For exam- 
ple, the models of Osram and Cree LEDs are available in the appropriate formats for the following programs: Zemax, TracePro, LightTools, etc.

\section{Overview of optical modeling tools}

The use of various software systems to automate the design process of lighting devices and systems is obvious in practice $[3,4]$. The following programs can be used as the tool for optical systems and programs calculation and development, the most common of which are Zemax, Light Tools, ASAP, TraceRro, OSLO. These systems provide end-to-end modeling, that is, the propagation of light from sources to radiation receivers.

Zemax program is the most popular one among developers. Its peculiarity is the following one: it combines the algorithms and the means of analysis for a consistent and an inconsistent calculation of rays. Zemax contains a huge database concerning the characteristics of various types of glass. This program is able to calculate the most complex construction of lens, mirror, diffraction grating, interference and absorption filter systems and other elements.

The developers of optical systems know well the program LightTools with a full set of opportunities for lighting system design, based on three-dimensional solid-state graphics. It allows you to build a lighting system model with a light source and its constituent optical and mechanical parts. During the calculation of rays, all possible light transformations are taken into account (refraction, reflection, total internal reflection, scattering, diffraction, and others). There are two approaches to analyze the illumination in LightTools: direct ray tracing, which simulates the propagation of light through a model, and reverse ray tracing, which shows an illumination source from a receiver point (in a reverse direction).

TracePro is a budget universal program for the calculation of rays during the design of lighting and non-reflective optical systems, as well as for the conduct of photometric analysis. This program offers three-dimensional solid visual imaging, the extensive opportunities for the analysis of optical parameters and the means for data exchange with CAD systems. It is possible to model the optical processes in the program based on the optical-mechanical models created in the CAD software SolidWorks, KOMPAS, etc., which significantly reduces developer's costs.

When you model the optical elements of lighting systems it is often necessary to work with light-scattering microstructures (a set of geometric elements applied to an optical system surface). The possibility of body modeling with the volume scattering is present in most optical modeling programs, where empirical models are used as dispersion models.

So, there is a large catalog of various transparent materials in Zemax for which all optical properties are determined already and do not require an additional task. TracePro and 
LightTools have special modeling tools for light scattering on microparticles. As a rule, such scattering micro-objects are called three-dimensional textures and allow to use different types of micro-element packages (regular or irregular ones). Regular microstructures determine the topography of a surface completely and represent a number of microgeometric elements distributed along an optical system surface according to some law.

\section{Modeling of LEDs and LED modules in TracePro software complex}

Perhaps nowadays, LEDs are the most promising sources of light, and they displace not only incandescent lamps, but also quite young luminescent lamps from many fields of use because of their uniqueness $[5,6]$.

The development of a lighting product begins with the creation of a model that must correspond to the parameters of a future product as precisely as possible. To do this it is necessary to develop a 3D model, create a database of optical properties for used materials and coatings. As it was noted, TracePro software complex allows to create three-dimensional models of optical-mechanical systems and conduct the analysis of the light distribution, taking into account light scattering, reflection, absorption and diffraction. This program performs ray tracing using Monte Carlo method, supports export/import to all known 3D formats and the import from Zemax, CodeV, Oslo. It is compatible with widely used CAD systems.

TracePro has the possibility to specify the optical properties of object surface materials and coatings. This facilitates the development, since it makes possible to use the optical properties of the material for an optical model of a part and assign the optical properties of coatings to its individual surfaces without the creation of separate objects. In order to create the model of an applied light source in accordance with the actual dimensions, the distribution of light flux, the spectral characteristics of the light emitted by it in TracePro, such an opportunity is present in an interactive mode.

The concept of a source file provides the possibility of an obtained light source distribution use or downloaded from TracePro site. You can also carry out the tracing using previously obtained data, create a source from other theoretical or measured data in another application, to use the obtained rays as a source in another model.

So, the dimensions of the 3D model from the manufacturer's site and the output data were used as an example for Siemens LED simulation in accordance with the photometric curve. The process of a model building consists of the following steps.

1. The design of the LED housing (or the LEDs in a LED module). To do this, you can build it yourself, or use the model created in other systems (for example, in SolidWorks) or use ready-made files provided by developers. 
2. Determination of optical properties.

3. Specification of LED characteristics.

4. Specification of ray tracing parameters (Fig. 1).

5. The selection of light distribution curve design option to obtain light intensity curve (Fig. 2).

6. The integration of an obtained model into the body of a reflector model and the analysis of this system (Fig. 3).

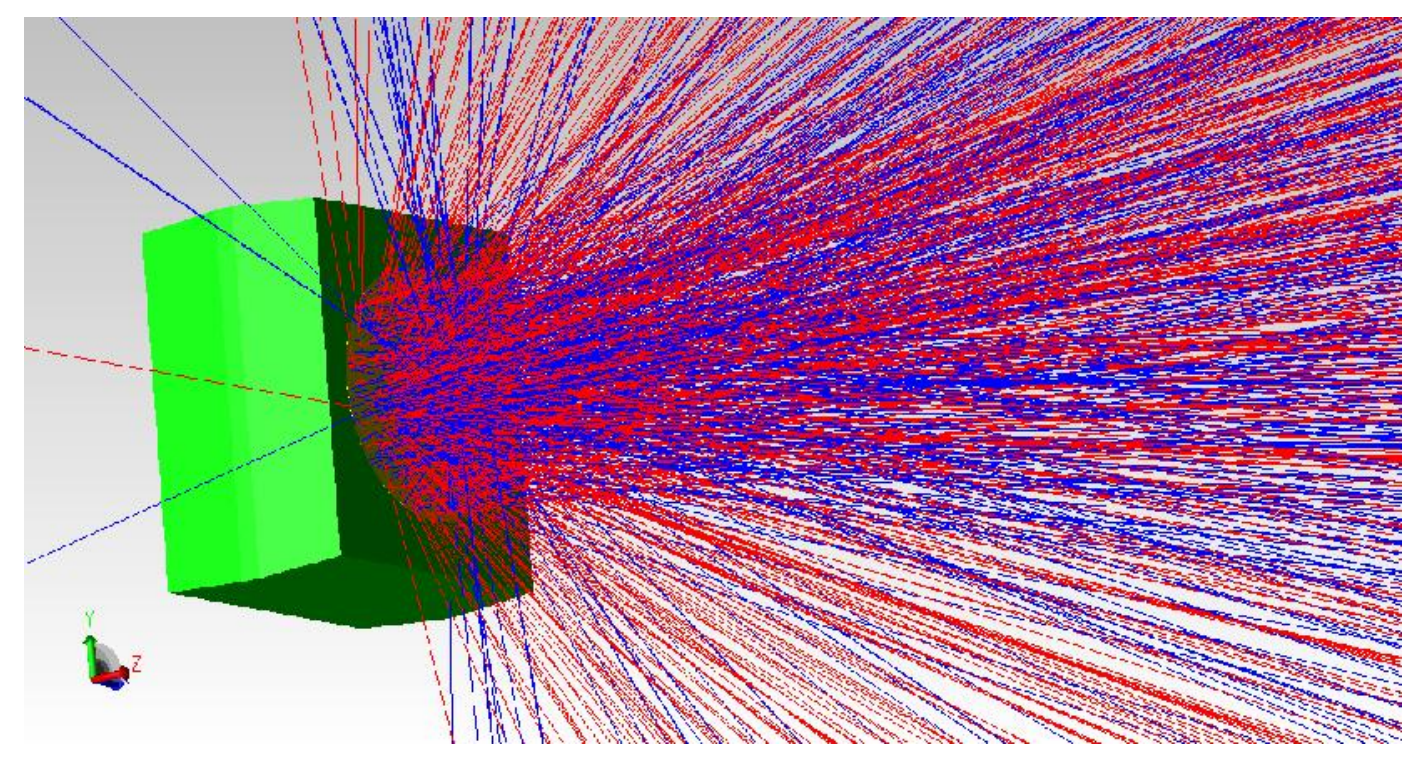

Fig.1. Beam tracing of Siemens LED
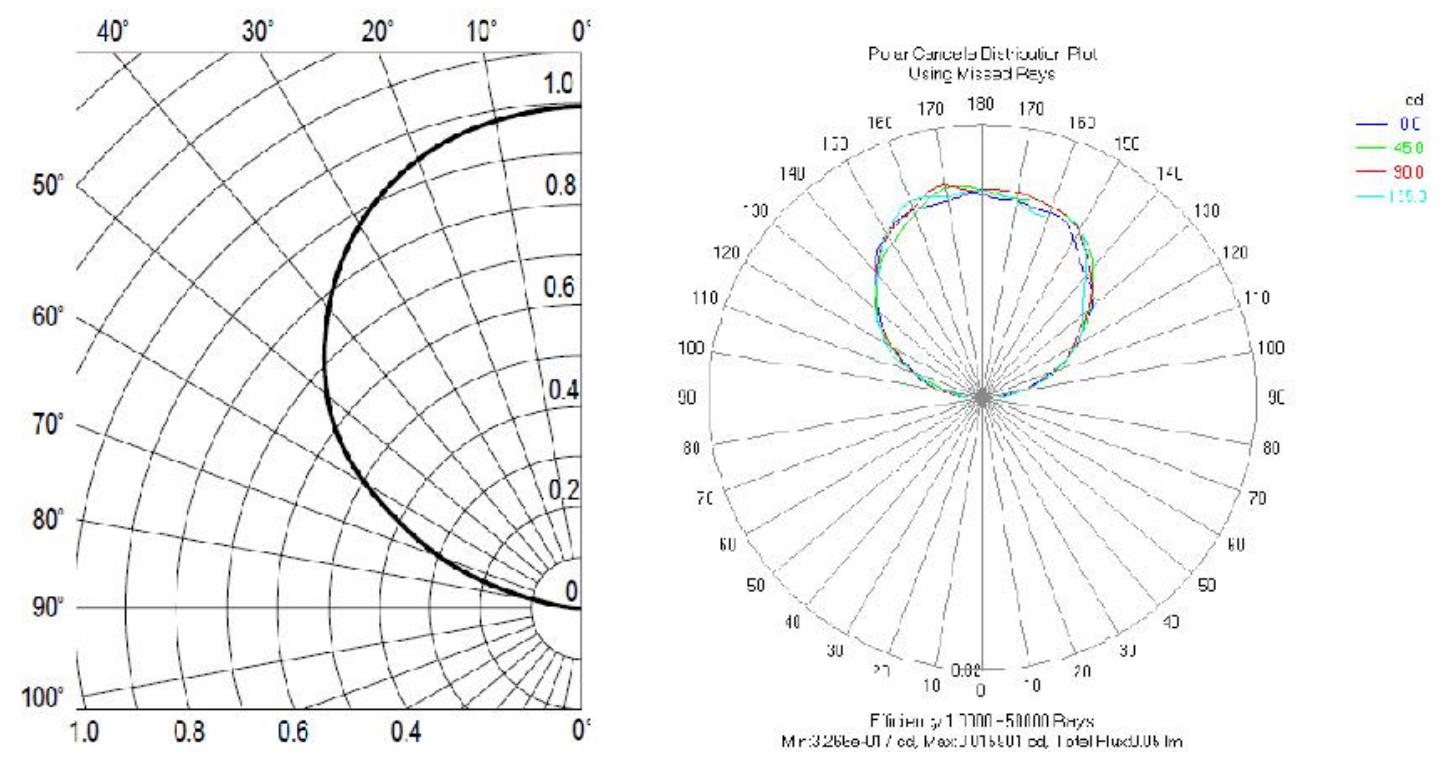

6

Fig.2. Curves of real (a) and simulated (b) light intensity of LED 


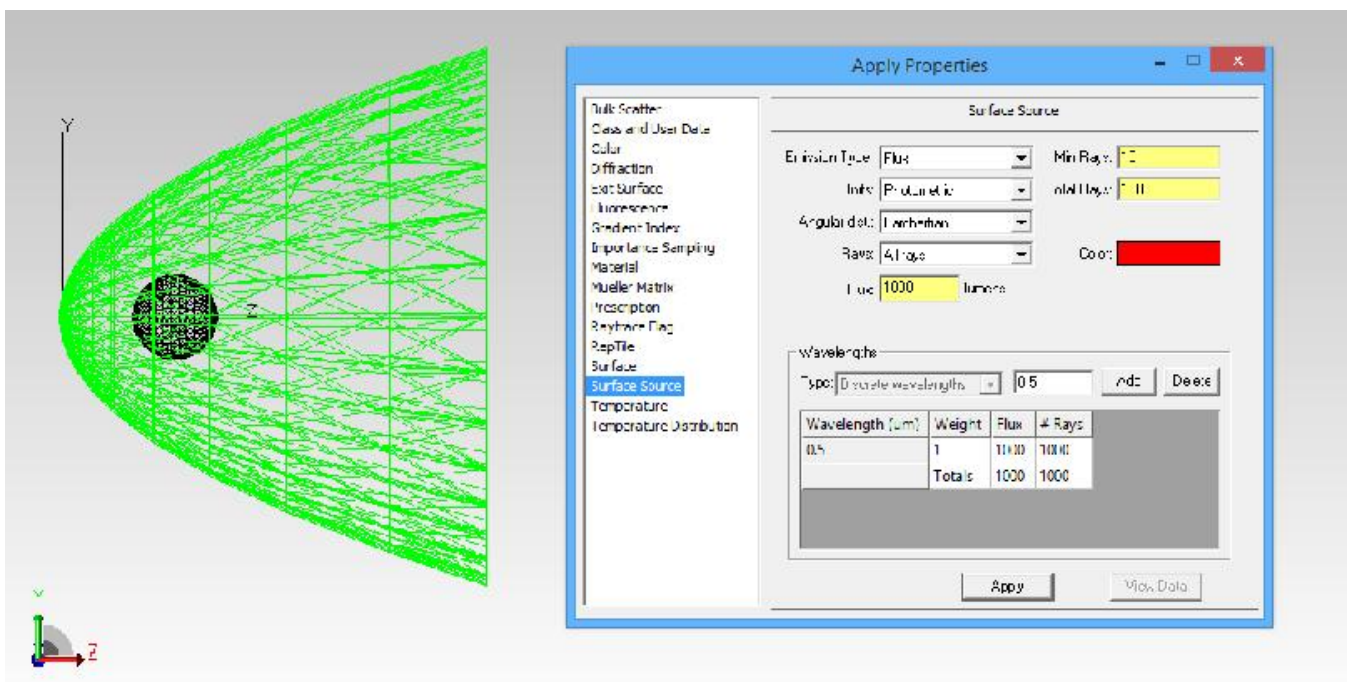

Fig.3. The frame model of the reflector with a light source

\section{The use of TracePro software complex in educational and scientific activities of masters}

"Computer Design and Lighting Device Design" discipline is taught at the Institute of Electronics and Lighting equipment of FSBEI HE "SI MSU named after N.P. Ogaryev" within the framework of the main educational program of higher education in the field of training 11.04.04 Electronics and nanoelectronics (magistracy) according to the profile of "Theoretical and Applied Lighting Equipment". Its laboriousness makes 3 credits (108 hours), 36 hours of which are laboratory studies. The discipline covers the range of issues related to the development of knowledge in the field of typical units and the elements of lighting devices, lamps of various production and purpose design, theories and bases for the calculation and design of lighting devices, including the use of modern software.

During the laboratory classes, the masters perform laboratory works on the construction of an optical and a structural part of light devices in the automated design system KOMPAS-3D. They also simulate the optical elements of lighting systems (for example, LEDs and LED modules (Figure 4-5) with different optical properties) [7-9] using TracePro software package. This contributes to the acquisition of a valuable working experience with modern software products and allows you to apply the gained knowledge gained carrying out research and the work in respect of a master's thesis.

\section{Summary}

Thus, it is advisable to use modern software with optimal functional properties and integration capabilities with other automated systems during the research and development of lighting systems. An important aspect is the use of such a software complex to obtain the practical skills of lighting system modeling during the training of masters. 


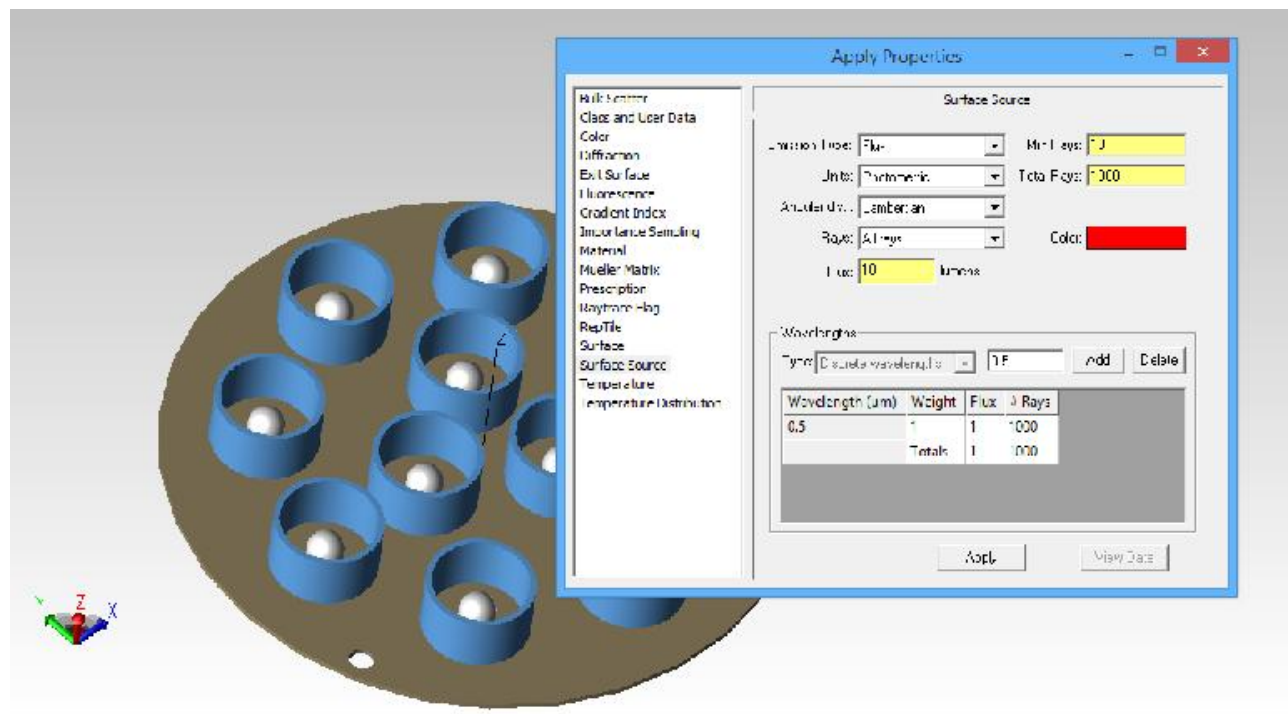

Fig.4. Modeling of the floodlight LED module in TracePro software package

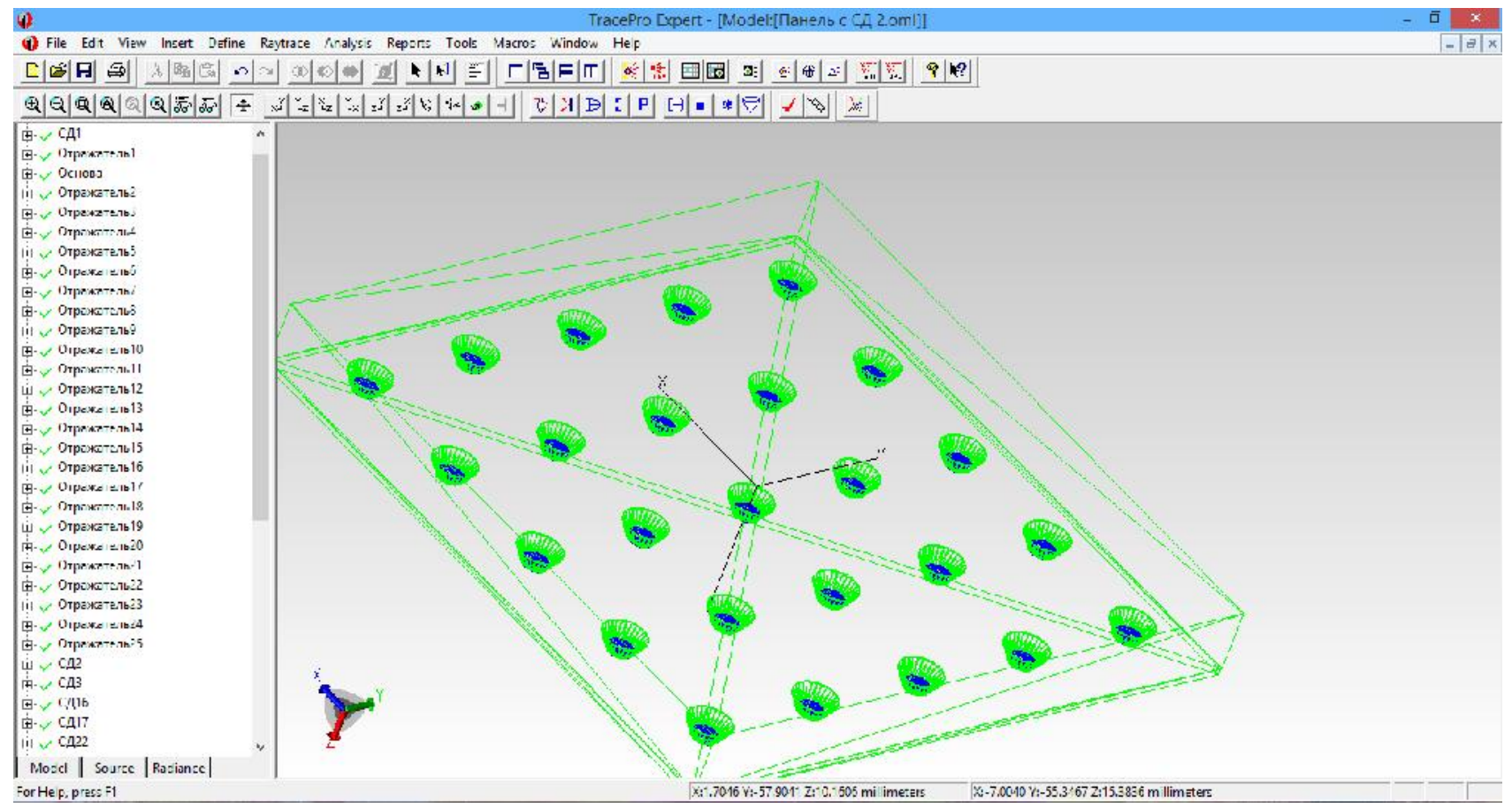

Fig.5. Modeling the LED module for ceiling light in TracePro software package

\section{REFERENCES}

1. Bayneva I.I., Bainev V.V. Evaluation of LED light source and device efficiency and reliability // Photonics. - 2011. - No. 3. - pp. 66-68.

2. Bayneva I.I., Bainev V.V. The software model to assess the efficiency and reliability of LED light sources and devices // Semiconductor lightning equipment. - 2011, - V.3. - № 11. pp. 40-42. 
3. Bayneva I. I. Concerns Of Design Of The Energy-Efficient Fixtures // International Journal of Applied Engineering Research. Vol.10, Number 3 (2015), pp. 6479-6487.

4. Bayneva I.I., Bainev V.V. Automation of light device designing process // Automation and modern technologies. - 2014, - №2. - pp. 3-9.

5. Bayneva I. I. The features and prospects for the development of modern halogen light sources // Journal of Engineering and Applied Sciences. 2016, № 11(4), pp. 701-704.

6. Bayneva I.I., Bainev V.V. Optical systems for LEDs / Reference book. Engineering magazine with the application. 2017. No. 1 (238). pp. 54-64.

7. Bayneva I. I. Study and review of optical systems for light emitting diodes // International Journal of Pharmacy \& Technology. 2016, Vol. 8, Issue No.3, P. 15304-15309.

8. Bayneva I.I., Bainev V.V. Optical systems for light emitting diodes / Photonics. 2016. № 2(56). pp. 84-93.

9. Bayneva I.I., Bainev V.V. Application of optical modeling programs in educational and scientific activity / Assembly in mechanical engineering and tool engineering. 2015, №4(28). pp. 53-62.

\section{How to cite this article:}

Bayneva I I. Features of optical modeling in educational and scientific activity. J. Fundam. Appl. Sci., 2017, 9(1S), 41-48. 